\title{
DESEMPENHO DE ESCOLARES COM TRANSTORNO DE DÉFICIT DE ATENÇÃO E HIPERATIVIDADE EM TAREFAS METALINGUISTICAS E DE LEITURA
}

\section{Performance of students with Attention Deficit Disorder and Hyperactivity in metalinguistic and reading tasks}

\author{
Vera Lúcia Orlandi Cunha ${ }^{(1)}$, Cláudia da Silva (2), Maria Dalva Lourencetti (3), \\ Niura Aparecida de Moura Ribeiro Padula ${ }^{(4)}$, Simone Aparecida Capellini ${ }^{(5)}$
}

\begin{abstract}
RESUMO
Objetivos: comparar e caracterizar o desempenho de escolares com Transtorno de Déficit de Atenção e Hiperatividade (TDAH) em tarefas metalinguísticas e de leitura com escolares sem queixa de transtornos comportamentais e/ou de aprendizagem. Método: participaram vinte escolares do $4^{\circ}$ ao $8^{\circ}$ ano do ensino fundamental, sendo catorze escolares do sexo masculino e seis do sexo feminino, na faixa etária de 9 a 13 anos. Os escolares foram divididos em dois grupos, sendo o Gl composto por dez escolares com diagnóstico interdisciplinar de TDAH e o Gll composto por dez escolares sem queixa de transtornos comportamentais e/ou de aprendizagem, pareados com os escolares do GI de acordo com a idade e o nível escolar. Foi utilizado como procedimento a aplicação de protocolo com provas de habilidades metalinguísticas e de leitura de palavras reais e pseudopalavras. Resultados: houve diferença estatisticamente significante entre os grupos nas tarefas de manipulação silábica e fonêmica, como também nas provas de leitura de palavras reais referentes à leitura de palavras regulares e palavras irregulares, bem como para as pseudopalavras. Conclusão: os escolares com Transtorno de Déficit de Atenção e Hiperatividade apresentaram desempenho inferior nas tarefas consideradas mais complexas, como a manipulação de sílabas e fonemas e na leitura de palavras irregulares, que exigem retenção, análise e recuperação de informação. As dificuldades apresentadas nessas habilidades pelos escolares com Transtorno de Déficit de Atenção e Hiperatividade podem ser atribuídas não a um déficit primário, mas como um fenômeno secundário à desatenção que interferem de forma direta em seu desempenho.
\end{abstract}

DESCRITORES: Transtorno do Déficit de Atenção com Hiperatividade; Leitura; Aprendizagem; Linguística; Educação

(1) Fonoaudióloga; Mestre e Doutoranda em Educação pela Faculdade de Filosofia e Ciências - FFC/UNESP, Marília, SP, Brasil.

(2) Fonoaudióloga; Mestre e Doutoranda em Educação pela Faculdade de Filosofia e Ciências - FFC/UNESP, Marília, SP, Brasil.

(3) Neuropsicóloga do Ambulatório de Desvios da Aprendizagem do HC/FM/UNESP, Botucatu, SP; Mestre em Saúde Coletiva pela Universidade do Sagrado Coração - USC, Bauru, SP, Brasil.

(4) Neurologista Infantil; Docente do Departamento de Neurologia e Psiquiatria da FM/UNESP - Botucatu - SP; Doutora em Ciências Médicas pela FCM/UNICAMP - Campinas, SP, Brasil.

\section{INTRODUÇÃO}

O Transtorno de Déficit de Atenção e Hiperatividade (TDAH) é atualmente considerado como um grupo heterogêneo de anormalidades neurológicas, que aparecem na infância e se manifestam durante

(5) Fonoaudióloga; Docente do Departamento de Fonoaudiologia e do Programa de Pós-Graduação em Educação da Faculdade de Filosofia e Ciências - FFC/UNESP - Marília - SP; Doutora em Ciências Médicas pela Faculdade de Ciências Médicas - FCM/UNICAMP, Campinas, SP, Brasil.

Conflito de interesses: inexistente 
o desenvolvimento, resultantes da interação entre determinadas configurações genéticas e exposição a várias condições ambientais. As classificações atuais de diagnóstico colocam essa heterogeneidade em uma categoria concebida como um grupo de anormalidades do desenvolvimento do lóbulo frontal e outras áreas cerebrais relacionadas ${ }^{1,2}$.

O TDAH pode ser entendido, portanto, como um transtorno dependente de fatores genéticofamiliares, adversidades biológicas e psicossociais. Tem etiologia multifatorial e caracteriza-se pela presença de um desempenho inapropriado nos mecanismos que regulam a atenção, a reflexibilidade e a atividade motora. Tem início precoce, com tendência a evolução crônica, apresentando repercussões significantes no funcionamento do sujeito em diversos contextos de sua vida ${ }^{3-5}$.

O TDAH é o distúrbio neuropsiquiátrico mais comum da infância e está incluído entre as doenças crônicas mais prevalentes entre escolares. Em amostras não referidas, estima-se que de $3 \%$ a $6 \%$ das crianças em idade escolar apresentam TDAH'. A dificuldade de sustentar a atenção é um dos sintomas mais prevalentes nas crianças que apresentam esse transtorno. Socialmente, tais crianças, muitas vezes, são percebidas como indisciplinadas, pela própria dificuldade de atentar e de seguir regras, visto ser o TDAH um transtorno do desenvolvimento do autocontrole que afeta a atenção, o controle de impulsos e o nível de atividade ${ }^{5,7,8}$.

Dessa forma, é no contexto escolar que a inquietude e a impulsividade são interpretadas como falta de disciplina e a desatenção, como negligência, já que esses sintomas do TDAH são possíveis de serem observados desde cedo, ainda em fases anteriores à pré-escola ${ }^{8,9}$. As dificuldades de atenção e de hiperatividade apresentadas por essas crianças podem comprometer seu desempenho acadêmico, pois o déficit de linguagem apresentado pode comprometer a aprendizagem do sistema de escrita alfabético, haja vista que habilidades subjacentes a esse processo, como as habilidades metalinguísticas, prejudicam essa aquisição ${ }^{10,11}$.

Vários estudos que estabeleceram uma relação entre TDAH e a aprendizagem da linguagem escrita relataram que os problemas de aprendizagem mais prevalentes afetam a área da leitura (8-39\%) e a escrita $(60 \%)^{3,5,7,8,12-14}$. Esses estudos permitiram postular hipóteses de que os déficits de linguagem em crianças com TDAH estão muito provavelmente relacionados a atividades cognitivas supraordenadas por comportamentos organizados que incluem aspectos da fala. Essas atividades podem ser denominadas em seu conjunto de funções executivas e incluem o estabelecimento de metas, programação, iniciação, controle, inibição de interferências, fluência, velocidade, organização temporal, sequencialização, comparação, classificação e categorização, as quais estão associadas com os sistemas corticais e subcorticais dos lóbulos frontais $7,15,16$.

Sendo as funções cognitivas estruturadas por múltiplos componentes, que podem ser específicos para algumas funções ou ter associações entre si, funções como a aquisição da linguagem e da aprendizagem escolar podem estar comprometidas em diversas faixas etárias do desenvolvimento da criança com o transtorno de TDAH. Deve-se, portanto, considerar que esse transtorno tem uma base neurobiológica ocasionando alterações em mecanismos cognitivos que estão associados com a aprendizagem, tais como atenção sustentada, funções executivas, déficit de inibição motora, entre outros $^{4-6,9,11,16,17}$. Além disso, levando-se em conta que as aquisições posteriores, como a aquisição da leitura e da escrita em um sistema de base alfabético, depende de aspectos subjacentes à linguagem oral como um bom desenvolvimento das habilidades metalinguísticas na fase pré-escolar, pode ser esperado que os déficits em linguagem apresentado por tais crianças têm forte relação com os déficits acadêmicos ${ }^{4,14,18,19}$.

Para a realização de atividades como leitura de palavras isoladas ou texto, é necessário um processamento visual refinado dos sinais gráficos para que se dê a realização de varredura textual para identificação das partes constituintes da palavra, sendo necessário considerar que esse processamento visual está relacionado com o processamento linguístico da leitura, que realiza a identificação da palavra mediante o processo de decodificação fonológica. Tal processo permite a conversão dos sinais gráficos em representações fonológicas. Crianças que apresentam falhas atencionais ou de processamento da informação terão dificuldade para acionar um processamento visual refinado, o que comprometerá o acesso fonológico exigido para a realização da leitura e escrita de um sistema alfabético ${ }^{5,19-22}$.

Considerando os aspectos expostos, este artigo teve por objetivos comparar e caracterizar o desempenho de escolares com TDAH em tarefas metalinguísticas e de leitura com escolares sem queixa de transtornos comportamentais e/ou de aprendizagem.

\section{MÉTODO}

Participaram deste estudo vinte escolares do $4^{\circ}$ ao $8^{\circ}$ ano do ensino fundamental, sendo catorze escolares do sexo masculino e seis do feminino, na faixa etária de 9 a 13 anos. Os escolares foram 
divididos em dois grupos, conforme descrito a seguir:

Grupo I (GI): composto por dez escolares com diagnóstico interdisciplinar de TDAH. O diagnóstico de TDAH desses escolares foi realizado por equipe interdisciplinar do Laboratório de Investigação dos Desvios da Aprendizagem do Centro de Estudos da Educação e Saúde - CEES/UNESP - Marília, incluindo avaliação fonoaudiológica, neurológica, neuropsicológica e seguindo critérios propostos pelo DSM-IV ${ }^{23}$. Todos os escolares desse grupo faziam uso de medicação por pelo menos seis meses antes da avaliação.

Grupo II (GII): composto por dez escolares sem queixa de transtornos comportamentais e/ou de aprendizagem. Esses escolares foram pareados com os escolares do Gl de acordo com a idade e a série escolar.

Os escolares de ensino público municipal do grupo Gll foram indicados por seus professores seguindo o critério de desempenho acadêmico satisfatório em dois bimestres consecutivos em avaliações de Língua Portuguesa e Matemática. A partir dessa indicação, os escolares foram submetidos previamente às avaliações otorrinolaringológica, audiológica e oftalmológica e somente participaram desse estudo aqueles que apresentaram resultados dentro dos padrões de normalidade.

A classificação do nível socioeconômico foi realizada com base no estudo estatístico do Índice de Desenvolvimento Socioeconômico - IDESE ${ }^{24}$, garantindo, assim, a homogeneidade da amostra do ponto de vista socioeconômico.

Foi considerado como critério de inclusão para o Grupo I que os escolares apresentassem ausência de queixa auditiva ou visual descritas nos prontuários escolares ou observação do professor e, para o Grupo II, que os escolares apresentassem diagnóstico interdisciplinar de TDAH há pelo menos seis meses.

Como critério de exclusão, foram consideradas a não assinatura dos pais ou responsáveis do Termo de Consentimento Livre e Esclarecido, conforme resolução do Conselho Nacional de Saúde CNS 196/96, a ausência de deficiência sensorial, motora ou cognitiva referida em prontuário escolar ou observação do professor.

Como procedimento, realizou-se a aplicação das provas metalinguísticas e de leitura do $\mathrm{PROH}$ MELE (Protocolo de Provas de Habilidades Metalinguísticas e de Leitura ${ }^{25}$, descritas a seguir:

Provas de identificação silábica e fonêmica: Identificação de Sílaba Inicial (ISI), Identificação de Fonema Inicial (IFI), Identificação de Sílaba Final (ISF), Identificação de Fonema Final (IFF), Iden- tificação de Sílaba Medial (ISM), Identificação de Fonema Medial (IFM).

Provas de manipulação silábica e fonêmica: Segmentação de Sílabas (Seg Sil), Segmentação de Fonemas(Seg Fon), Adição de Sílabas (Ad Sil), Adição de Fonemas (Ad Fon), Substituição de Sílabas (Subs Sil), Substituição de Fonemas (Subs Fon), Subtração de Sílabas (Subt Sil), Subtração de Fonemas (Subt Fon), Combinação de Sílabas (Com Sil), Combinação de Fonemas (Com Fon).

Provas de leitura: Leitura de Palavras Reais: foi apresentada lista de palavras reais isoladas (133 palavras); Leitura de Pseudopalavras: foi apresentada lista de pseudopalavras (27 pseudopalavras).

As pseudopalavras são entendidas, aqui, como logatomo, ou seja, uma sílaba ou uma sequência de sílabas que pertencem à língua, mas que não formam uma palavra com significado ${ }^{26}$. A pseudopalavra é derivada de uma palavra real, como, por exemplo, "bafata", derivada de "barata", mudandose apenas um elemento e mantendo-se o padrão silábico.

A aplicação das provas de habilidades metalinguísticas foi realizada de forma que o escolar não tivesse pista visual da articulação dos sons produzidos pela examinadora. As respostas do escolar foram anotadas na folha de respostas do $\mathrm{PROH}$ MELE. O escolar foi instruído e treinado previamente por meio de exemplos similares aos da prova para que soubesse o que deveria fazer.

As provas de leitura foram realizadas em voz alta e gravadas em gravador digital para posterior análise da leitura. Cada escolar recebeu instrução de como deveria ler as listas de palavras, apresentadas no formato de letra arial tamanho 14, espaço duplo, divididas em colunas segundo extensão de palavras (monossilábicas, dissilábicas, trissilábicas e polissilábicas - de 4 a 7 sílabas) e de pseudopalavras (monossilábicas, dissilábicas, trissilábicas). Na prova de leitura de pseudopalavras, foi esclarecido aos escolares que iriam ler palavras que não existem e que, por isso, não fazem parte de seu vocabulário. As provas foram aplicadas individualmente em uma sessão com cerca de 50 minutos.

Todas as provas deste estudo foram analisadas segundo o critério de erros. A caracterização dos tipos de erros da leitura de palavras reais e pseudopalavras foi realizada a partir de critérios estabelecidos para o português do $\mathrm{Brasil}^{26}$, descritos a seguir:

- D1 - Regra de correspondência grafofonêmica independente do contexto referentes a palavras regulares com correspondência unívoca. Em palavras como: pato, bolo, faca, vela, tatu, dado, massa, moça, desço, chuva, janela, 
unha, carro, óculos, põe, água, lâmpada, rã, etc.

- D2 - Regra de correspondência grafofonêmica dependente do contexto referentes às regras aplicadas às palavras irregulares. Como, por exemplo: casa, zebra, costa, piscina, árvore, rato, cara, ganso, bolsa, homem, galho, gente, cinema, guarda, guizo, leque, quadro, exceção, xícara, exame, tórax, caixa, enxame, etc.

- D4 - Valores da letra "X" dependentes exclusivamente do léxico mental e ortográfico, que aprecem em palavras como: abacaxi, táxi, oxigênio, auxílio, próximo, etc.,

Na prova de leitura de pseudopalavras, foi considerada somente a regra $\mathrm{D} 1$, pois seu objetivo é verificar a correspondência unívoca entre letra e som.

Os escolares do Gl foram avaliados após 30 minutos da administração do medicamento (metilfenidato), uma vez que na ausência da medicação não foi possível realizar a avaliação proposta neste estudo. Foram realizadas duas sessões para a avaliação com duração de 30 minutos cada. E os escolares do Gll foram avaliados em uma sessão em uma sala de aula fornecida pela coordenação pedagógica, em horário predeterminado pelo professor de cada escolar.

Este trabalho foi aprovado pelo Comitê de Ética em Pesquisa da Faculdade de Filosofia e Ciências da Universidade Estadual Paulista - FFC/UNESP/ Marília - SP, sob o protocolo de número 3326/2006.

Foi adotado o nível de significância de 5\% $(0,050)$ para a aplicação dos testes estatísticos, ou seja, quando o valor da significância calculada (p) for menor do que $5 \%(0,050)$ observou-se uma diferença ou uma relação dita "estatisticamente significante" (marcada em asterisco). O programa utilizado foi o SPSS (Statistical Package for Social Sciences), em sua versão 19.0, para a obtenção dos resultados.

\section{RESULTADOS}

Após a análise estatística dos dados por meio da aplicação do Teste de Mann-Whitney, com o intuito de verificar possíveis diferenças entre ambos os grupos estudados para as variáveis de interesse, foi observado, como mostra a Tabela 1, que nas provas de identificação de sílabas e fonemas não houve significância entre os grupos nas variáveis estudadas.

Tabela 1 - Comparação entre GI e GII nas provas de identificação de sílabas e fonemas

\begin{tabular}{|c|c|c|c|c|c|c|}
\hline Variável & Grupo & Média & $\begin{array}{l}\text { Desvio- } \\
\text { padrão }\end{array}$ & Mínimo & Máximo & $\begin{array}{l}\text { Significância } \\
\text { (p) }\end{array}$ \\
\hline \multirow{2}{*}{ ISI } & 1 & 0,40 & 1,27 & 0,00 & 4,00 & \multirow{2}{*}{0,317} \\
\hline & ॥ & 0,00 & 0,00 & 0,00 & 0,00 & \\
\hline \multirow{2}{*}{ IFI } & 1 & 0,20 & 0,42 & 0,00 & 1,00 & \multirow{2}{*}{0,626} \\
\hline & II & 0,20 & 0,63 & 0,00 & 2,00 & \\
\hline \multirow{2}{*}{ ISF } & 1 & 0,10 & 0,32 & 0,00 & 1,00 & \multirow{2}{*}{0,503} \\
\hline & II & 0,40 & 0,97 & 0,00 & 3,00 & \\
\hline \multirow{2}{*}{ IFF } & 1 & 1,00 & 1,89 & 0,00 & 5,00 & \multirow{2}{*}{0,234} \\
\hline & II & 0,10 & 0,32 & 0,00 & 1,00 & \\
\hline \multirow{2}{*}{ ISM } & 1 & 0,60 & 1,27 & 0,00 & 4,00 & \multirow{2}{*}{0,068} \\
\hline & II & 0,00 & 0,00 & 0,00 & 0,00 & \\
\hline \multirow{2}{*}{ IFM } & 1 & 1,40 & 1,84 & 0,00 & 5,00 & \multirow{2}{*}{0,325} \\
\hline & II & 0,70 & 1,34 & 0,00 & 4,00 & \\
\hline
\end{tabular}

Legenda: ISI: identificação de sílaba inicial; IFI: identificação de fonema inicial; ISF: identificação de sílaba final; IFF: identificação de fonema final; ISM: identificação de sílaba medial; IFM: identificação de fonema medial Análise estatística: Teste de Mann-Whitney p-valor: > 0,050 
$\mathrm{Na}$ Tabela 2, observou-se que nas provas de manipulação de sílabas e fonemas houve significância (marcadas com asterisco na tabela) entre os grupos em Segmentação Fonêmica, Adição Silábica, Adição Fonêmica, Substituição Silábica,
Substituição Fonêmica, Combinação Silábica e Combinação Fonêmica, indicando que nessas variáveis o grupo Gll apresentou desempenho superior ao grupo $\mathrm{Gl}$.

Tabela 2 - Comparação entre GI e GII nas provas de manipulação de sílabas e fonemas

\begin{tabular}{|c|c|c|c|c|c|c|}
\hline Variável & Grupo & Média & $\begin{array}{l}\text { Desvio- } \\
\text { padrão }\end{array}$ & Mínimo & Máximo & $\begin{array}{c}\text { Significância } \\
\text { (p) }\end{array}$ \\
\hline \multirow{2}{*}{ Seg Sil } & 1 & 0,30 & 0,48 & 0,00 & 1,00 & \multirow{2}{*}{$>0,999$} \\
\hline & II & 0,30 & 0,48 & 0,00 & 1,00 & \\
\hline \multirow{2}{*}{ Seg Fon } & 1 & 7,60 & 3,98 & 0,00 & 10,00 & \multirow{2}{*}{$0,004^{*}$} \\
\hline & II & 1,50 & 1,27 & 0,00 & 4,00 & \\
\hline \multirow{2}{*}{ Ad Sil } & 1 & 2,10 & 3,18 & 0,00 & 10,00 & \multirow{2}{*}{$0,040^{*}$} \\
\hline & II & 0,10 & 0,32 & 0,00 & 1,00 & \\
\hline \multirow{2}{*}{ Ad Fon } & 1 & 7,30 & 3,89 & 0,00 & 10,00 & \multirow{2}{*}{$<0,001^{*}$} \\
\hline & II & 0,00 & 0,00 & 0,00 & 0,00 & \\
\hline \multirow{2}{*}{ Subs Sil } & 1 & 4,70 & 4,72 & 0,00 & 10,00 & \multirow{2}{*}{$0,005^{\star}$} \\
\hline & II & 0,10 & 0,32 & 0,00 & 1,00 & \\
\hline \multirow{2}{*}{ Subs Fon } & 1 & 6,20 & 4,92 & 0,00 & 10,00 & \multirow{2}{*}{$0,004^{*}$} \\
\hline & II & 0,30 & 0,68 & 0,00 & 2,00 & \\
\hline \multirow{2}{*}{ Subt Sil } & 1 & 1,60 & 1,78 & 0,00 & 4,00 & \multirow{2}{*}{0,199} \\
\hline & II & 0,60 & 1,08 & 0,00 & 3,00 & \\
\hline \multirow{2}{*}{ Subt Fon } & I & 4,90 & 4,86 & 0,00 & 10,00 & \multirow{2}{*}{0,104} \\
\hline & II & 0,80 & 1,14 & 0,00 & 3,00 & \\
\hline \multirow{2}{*}{ Com Sil } & $\mathrm{I}$ & 2,20 & 1,87 & 0,00 & 5,00 & \multirow{2}{*}{$0,028^{*}$} \\
\hline & II & 0,50 & 1,27 & 0,00 & 4,00 & \\
\hline \multirow{2}{*}{ Com Fon } & 1 & 7,30 & 4,42 & 0,00 & 10,00 & \multirow{2}{*}{$0,012^{*}$} \\
\hline & II & 1,20 & 1,23 & 0,00 & 4,00 & \\
\hline
\end{tabular}

Legenda: Seg Sil: segmentação silábica; Seg Fon: segmentação fonêmica; Ad Sil: adição silábica; Ad Fon: adição fonêmica; Subs Sil: substituição silábica; Subs Fon: substituição fonêmica; Subt Sil: subtração silábica; Subt Fon: subtração fonêmica; Com Sil: combinação de sílabas; Com Fon: Combinação de fonemas

Análise estatística: Teste de Mann-Whitney

p-valor: $>0,050$

Nas provas de leitura de palavras reais e de pseudopalavras, descritas na tabela 3, houve diferença estatisticamente significante (marcadas com asterisco na tabela) para as palavras referentes à regra D1, que são as palavras regulares, com correspondência grafofonêmica independente do contexto.

A tabela 4 mostra os resultados da leitura de palavras reais para as regras de D2.1 a D2.11, correspondentes às palavras irregulares, com correspondência grafofonêmica dependente do contexto. Nessas regras observou-se que houve diferença estatisticamente significante (marcadas com asterisco na tabela) entre os grupos somente para as regras D2.3 e D2.5. A regra D2.3 refere-se à leitura do grafema "s" em final de sílaba interna; em sua realização copia o traço sonoro se a letra seguinte representar uma consoante sonora e copia o traço [-son] se ela representar uma consoante surda, em palavras como: festa, hospital, 
Tabela 3 - Comparação entre os grupos GI e Gll para as regras D1 na leitura de palavras reais e pseudopalavras

\begin{tabular}{ccccccc}
\hline Variável & Grupo & Média & $\begin{array}{c}\text { Desvio- } \\
\text { padrão }\end{array}$ & Mínimo & Máximo & $\begin{array}{c}\text { Significância } \\
(\mathbf{p})\end{array}$ \\
\hline \multirow{2}{*}{ D1 PR } & I & 8,80 & 14,18 & 0,00 & 45,00 & $0,024^{*}$ \\
\hline \multirow{2}{*}{ D1PP } & II & 0,44 & 0,88 & 0,00 & 2,00 & $0,007^{*}$ \\
\hline
\end{tabular}

Legenda: D1 PR: regra de correspondência grafofonêmica independente do contexto para palavras reais; D1 PP: regra de correspondência grafofonêmica independente do contexto para pseudopalavras

Análise estatística: Teste de Mann-Whitney

p-valor: $>0,050$

descanso, responsabilidade. A regra D2.5 refere-se à leitura do grafema "z" em início de vocábulo e em início de sílaba; antes de letras que representam vogais é transposto à realização do fonema /z/, em palavras como: zoo, zero, horizonte. O grupo Gll apresentou desempenho superior em relação ao grupo Gl.

A tabela 5 mostra os resultados da leitura de palavras reais para as regras de D2.12 a D2.23, correspondentes às palavras irregulares, com correspondência grafofonêmica dependente do contexto. Observou-se que houve diferença estatisticamente significante (marcadas com asterisco na tabela) entre os grupos somente para a regra D2.23, que se refere à regra correspondente à leitura dos grafemas "i" e "u"; leem-se, respectivamente, como a realização das semivogais /j/ e /w/, quando ocorrerem seguidos ou não de "s", depois de vogal na mesma sílaba (ditongo decrescente), em final de vocábulo e em sílaba interna; em final de sílaba interna, a ocorrência de "s" é marginal; como a realização das semivogais /j/ e / $\mathrm{w} /$, quando ocorrerem antes de vogal, seguida ou não de consoante, na mesma sílaba (ditongo crescente); como a realização de /i/ e /u/, respectivamente, se estiverem antes de letra, na mesma sílaba, que não "m" ou " $n$ ". Aparecem em palavras como: luz, sul, boi, água, caixa, quadro, lâmina, fábula, urgência, guerreiro, auxílio. O grupo Gll apresentou desempenho superior em relação ao grupo $\mathrm{Gl}$.

A tabela 6 mostra os resultados da leitura de palavras reais para a regra D4, referente a três valores atribuídos à letra " $x$ " que dependem exclusivamente da internalização do léxico mental ortográfico e de suas relações com o léxico mental fonológico, sendo "/x/", /s/ e /k(i)s/ quando no contexto entre letras que representam vogais (com exceção da letra "e" em início de vocábulo, precedida ou não por prefixo), e entre o ditongo /aw/ e vogal. A letra " $x$ ", entre letras que representam vogais, se a segunda vogal começar a terminação "imu" -/imo/ ou "im" -/imi/ e suas reflexões, lê-se como a realização do fonema /s/. Aparecem em palavras como: táxi, tóxico, próximo. Observou-se que houve diferença estatisticamente significante (marcadas com asterisco na tabela) entre os grupos, com desempenho superior para o grupo Gll.

\section{DISCUSSÃO}

De acordo com os resultados obtidos neste estudo, é possível verificar que a média do desempenho do grupo Gl, ou seja, dos escolares com diagnóstico de TDAH, foi estatisticamente inferior ao desempenho do grupo Gll em todas as habilidades metalinguísticas avaliadas, havendo diferença estatisticamente significante em provas de manipulação fonêmica e silábica, como segmentação, adição, substituição e combinação, que requerem tempo de atenção e concentração mais apurados em relação às tarefas de identificação das sílabas e dos fonemas ${ }^{11,25,27}$.

Algumas tarefas de habilidades metalinguísticas, como a identificação de sílabas e fonemas, são consideradas mais simples, exigindo apenas uma operação seguida de resposta. Entretanto, tarefas mais complexas, como as de manipulação de sílabas e fonemas, exigem a realização de duas operações, ou seja, guardar uma unidade na memória enquanto é feita uma nova operação. Portanto, as respostas variam de acordo com o tipo de operação que é exigida da criança ${ }^{11,25,27}$.

Tais dados sugerem que o desempenho dos escolares com TDAH nessas habilidades podem ter sofrido interferência de fatores característicos do próprio diagnóstico, como a desorganização, a hiperatividade e a alteração atencional, interferindo de forma direta na retenção da informação, 
Tabela 4 - Comparação entre os grupos GI e GIl para as regras de D2.1 a D2.11 na leitura de palavras reais

\begin{tabular}{|c|c|c|c|c|c|c|}
\hline Variável & Grupo & Média & $\begin{array}{l}\text { Desvio- } \\
\text { padrão }\end{array}$ & Mínimo & Máximo & $\begin{array}{c}\text { Significância } \\
\text { (p) }\end{array}$ \\
\hline \multirow{2}{*}{ D2.1 PR } & 1 & 0,44 & 1,01 & 0,00 & 3,00 & \multirow{2}{*}{0,586} \\
\hline & II & 0,33 & 1,00 & 0,00 & 3,00 & \\
\hline \multirow{2}{*}{ D 2.2} & 1 & 3,30 & 7,38 & 0,00 & 24,00 & \multirow{2}{*}{0,118} \\
\hline & II & 0,22 & 0,44 & 0,00 & 1,00 & \\
\hline \multirow{2}{*}{ D 2.3} & I & 1,30 & 2,11 & 0,00 & 6,00 & \multirow{2}{*}{$0,039^{*}$} \\
\hline & II & 0,00 & 0,00 & 0,00 & 0,00 & \\
\hline \multirow{2}{*}{ D 2.4} & 1 & 0,22 & 0,44 & 0,00 & 1,00 & \multirow{2}{*}{0,634} \\
\hline & II & 0,22 & 0,67 & 0,00 & 2,00 & \\
\hline \multirow{2}{*}{ D 2.5} & I & 0,70 & 0,95 & 0,00 & 2,00 & \multirow{2}{*}{$0,039^{*}$} \\
\hline & II & 0,00 & 0,00 & 0,00 & 0,00 & \\
\hline \multirow{2}{*}{ D 2.6.1 } & I & 0,60 & 1,08 & 0,00 & 3,00 & \multirow{2}{*}{0,330} \\
\hline & II & 0,22 & 0,67 & 0,00 & 2,00 & \\
\hline \multirow{2}{*}{ D 2.6.3 } & 1 & 1,20 & 2,10 & 0,00 & 6,00 & \multirow{2}{*}{0,741} \\
\hline & II & 0,78 & 1,39 & 0,00 & 4,00 & \\
\hline \multirow{2}{*}{ D 2.6.4 } & I & 0,60 & 0,52 & 0,00 & 1,00 & \multirow{2}{*}{0,768} \\
\hline & II & 0,63 & 0,92 & 0,00 & 2,00 & \\
\hline \multirow{2}{*}{ D 2.6.5 } & I & 0,00 & 0,00 & 0,00 & 0,00 & \multirow{2}{*}{$>0,999$} \\
\hline & II & 0,00 & 0,00 & 0,00 & 0,00 & \\
\hline \multirow{2}{*}{ D 2.7} & 1 & 1,30 & 3,13 & 0,00 & 10,00 & \multirow{2}{*}{0,068} \\
\hline & II & 0,00 & 0,00 & 0,00 & 0,00 & \\
\hline \multirow{2}{*}{ D 2.8} & I & 0,50 & 0,71 & 0,00 & 2,00 & \multirow{2}{*}{0,178} \\
\hline & II & 0,20 & 0,63 & 0,00 & 2,00 & \\
\hline \multirow{2}{*}{ D 2.9} & I & 0,50 & 1,07 & 0,00 & 3,00 & \multirow{2}{*}{0,104} \\
\hline & II & 0,00 & 0,00 & 0,00 & 0,00 & \\
\hline \multirow{2}{*}{ D 2.10} & I & 0,50 & 1,41 & 0,00 & 4,00 & \multirow{2}{*}{0,264} \\
\hline & II & 0,00 & 0,00 & 0,00 & 0,00 & \\
\hline \multirow{2}{*}{ D 2.11} & 1 & 1,56 & 4,30 & 0,00 & 13,00 & \multirow{2}{*}{0,126} \\
\hline & II & 0,00 & 0,00 & 0,00 & 0,00 & \\
\hline
\end{tabular}

Legenda: D2: Regra de correspondência grafofonêmica dependente do contexto para palavras reais

Análise estatística: Teste de Mann-Whitney. p-valor: > 0,050 
Tabela 5 - Comparação entre os grupos GI e Gll para as regras de D2.12 a D2.23 na leitura de palavras reais

\begin{tabular}{|c|c|c|c|c|c|c|}
\hline Variável & Grupo & Média & $\begin{array}{l}\text { Desvio- } \\
\text { padrão }\end{array}$ & Mínimo & Máximo & $\begin{array}{c}\text { Significância } \\
\text { (p) }\end{array}$ \\
\hline \multirow{2}{*}{ D 2.12} & 1 & 0,25 & 0,71 & 0,00 & 2,00 & \multirow{2}{*}{0,871} \\
\hline & II & 0,20 & 0,63 & 0,00 & 2,00 & \\
\hline \multirow{2}{*}{ D 2.13} & 1 & 2,00 & 4,40 & 0,00 & 14,00 & \multirow{2}{*}{0,213} \\
\hline & II & 0,10 & 0,32 & 0,00 & 1,00 & \\
\hline \multirow{2}{*}{ D 2.14} & $I$ & 0,11 & 0,33 & 0,00 & 1,00 & \multirow{2}{*}{$>0,999$} \\
\hline & II & 0,30 & 0,95 & 0,00 & 3,00 & \\
\hline \multirow{2}{*}{ D 2.15} & 1 & 2,20 & 5,94 & 0,00 & 19,00 & \multirow{2}{*}{0,234} \\
\hline & II & 0,10 & 0,32 & 0,00 & 1,00 & \\
\hline \multirow{2}{*}{ D 2.16} & 1 & 0,33 & 0,71 & 0,00 & 2,00 & \multirow{2}{*}{0,563} \\
\hline & II & 0,30 & 0,95 & 0,00 & 3,00 & \\
\hline \multirow{2}{*}{ D 2.17} & $I$ & 3,60 & 8,70 & 0,00 & 28,00 & \multirow{2}{*}{0,101} \\
\hline & II & 0,10 & 0,32 & 0,00 & 1,00 & \\
\hline \multirow{2}{*}{ D 2.18} & 1 & 0,25 & 0,71 & 0,00 & 2,00 & \multirow{2}{*}{0,717} \\
\hline & II & 0,22 & 0,44 & 0,00 & 1,00 & \\
\hline \multirow{2}{*}{ D 2.19.1 } & 1 & 0,30 & 0,68 & 0,00 & 2,00 & \multirow{2}{*}{0,914} \\
\hline & II & 0,40 & 0,84 & 0,00 & 2,00 & \\
\hline \multirow{2}{*}{ D 2.19 .2} & $I$ & 1,00 & 2,65 & 0,00 & 8,00 & \multirow{2}{*}{0,458} \\
\hline & II & 1,00 & 1,49 & 0,00 & 4,00 & \\
\hline \multirow{2}{*}{ D2.21 } & 1 & 0,70 & 1,57 & 0,00 & 5,00 & \multirow{2}{*}{0,618} \\
\hline & II & 0,30 & 0,68 & 0,00 & 2,00 & \\
\hline \multirow{2}{*}{ D 2.22} & 1 & 3,60 & 10,01 & 0,00 & 32,00 & \multirow{2}{*}{0,255} \\
\hline & II & 0,20 & 0,63 & 0,00 & 2,00 & \\
\hline \multirow{2}{*}{ D 2.23} & $I$ & 3,20 & 8,39 & 0,00 & 27,00 & \multirow{2}{*}{$0,049^{*}$} \\
\hline & II & 0,10 & 0,32 & 0,00 & 1,00 & \\
\hline
\end{tabular}

Legenda: D2: Regra de correspondência grafofonêmica dependente do contexto para palavras reais Análise estatística: Teste de Mann-Whitney

p-valor: $>0,050$

Tabela 6 - Comparação entre os grupos GI e GII para as regras D4 na leitura de palavras reais

\begin{tabular}{ccccccc}
\hline Variável & Grupo & Média & $\begin{array}{c}\text { Desvio- } \\
\text { padrão }\end{array}$ & Mínimo & Máximo & $\begin{array}{c}\text { Significância } \\
(\mathbf{p})\end{array}$ \\
\hline \multirow{2}{*}{ D4 } & I & 1,50 & 1,51 & 0,00 & 4,00 & $0,045^{\star}$ \\
\hline
\end{tabular}

Legenda: D4: valores da letra "X" dependentes exclusivamente do léxico mental e ortográfico

Análise estatística: Teste de Mann-Whitney

p-valor: > 0,050 
prejudicando o processamento da informação auditiva e visual, como apontam os estudos que atribuíram alterações de processamento auditivo não a um déficit primário, mas à hipótese de que crianças com TDAH apresentam essas alterações como um fenômeno secundário à desatenção que apresentam $^{11,18,28,29}$.

Nossos achados estão de acordo com o estudo ${ }^{30}$ cujos resultados indicaram que os sintomas da alteração de processamento auditivo coincidem com boa parte dos sintomas de TDAH. Dentre tais sintomas, podem ser destacados a dificuldade de atenção e escuta, má adaptação comportamental, facilidade em se distrair, dificuldades em seguir instruções e necessidade de um tempo maior que a média para completar tarefas, fatores que podem interferir na realização das tarefas metalinguísticas.

Os dados encontrados corroboraram estudos realizados anteriormente ${ }^{18,28}$ que apontaram que os aspectos da linguagem mais prejudicados nas crianças com transtorno de TDAH são o fonológico, o sintático e o pragmático. Esses autores, ao investigarem o componente semântico por meio de tarefas de organização semântica, memória auditiva e fluência verbal, constataram valores próximos aos dos sujeitos normais. Dessa forma, concluíram que as dificuldades para os aspectos fonético-fonológicos e gramaticais são causadas possivelmente por uma dificuldade de atenção e de controle inibitório dos estímulos irrelevantes do que por uma incapacidade específica para manejar os aspectos linguísticos.

Em relação às provas de leitura, foi possível observar que houve diferença estatisticamente significante nas provas envolvendo as regras D1, D2.3, D2.5, D2.23 e D2.4, ou seja, provas referentes à leitura de palavras reais e irregulares e provas que envolvem os valores da letra " $x$ ".

Os dados encontrados corroboraram estudos $^{20,21,31,32}$ que relataram que as alterações de leitura descritas no TDAH são decorrentes de uma desorganização sequencial e temporal dos fonemas necessários para a execução da atividade proposta, pois a presença de dificuldade no controle da seleção do segmento utilizado, no caso o fonema, em conjunto com a alteração atencional, leva à omissão de fonemas, sílabas e/ou palavras, resultando em uma leitura comprometida. Crianças com TDAH apresentam alterações de leitura e escrita decorrentes de déficits em memória de trabalho e consciência fonológica, logo a realização de provas que envolvem a leitura de palavras reais irregulares estará alterada, como ocorreu nos resultados encontrados no presente estudo.

Os resultados encontrados estão de acordo com pesquisa anterior ${ }^{11}$, que evidenciou que a prevalência das dificuldades de leitura em crianças com TDAH não é uma consequência de uma desordem de linguagem de base fonológica, mas sim uma consequência secundária dos problemas de autorregulação e de atenção inerente ao TDAH. Os autores argumentaram que, inicialmente, a decodificação de palavras demanda atenção e autorregulação, áreas que frequentemente são difíceis para as crianças com TDAH, por isso há uma forte associação entre TDAH e incapacidade de leitura.

\section{CONCLUSÃO}

Os achados deste estudo nos possibilitaram concluir que os escolares com TDAH não diferiram do grupo sem queixa de transtornos comportamentais e/ou de aprendizagem em tarefas consideradas mais simples, como a identificação de sílabas e fonemas e na leitura de palavras regulares. No entanto, apresentaram desempenho inferior nas tarefas consideradas mais complexas, como a manipulação de sílabas e fonemas e na leitura de palavras irregulares, que exigem retenção, análise e recuperação de informação. Dessa forma, as dificuldades apresentadas nessas habilidades pelos escolares com TDAH podem ser atribuídas não a um déficit primário, mas como um fenômeno secundário à desatenção que interferem de forma direta em seu desempenho.

\section{AGRADECIMENTOS}

À Coordenação de Pessoal de Nível Superior (Capes) e ao Conselho Nacional de Desenvolvimento Científico e Tecnológico (CNPq) pelo apoio à realização da pesquisa. 


\section{ABSTRACT}

Purposes: to compare and characterize the performance of students with Attention Deficit Hyperactivity Disorder (ADHD) in metalinguistic tasks and reading with students without behavioral disorders and/or learning disabilities. Method: twenty students from the $4^{\text {th }}$ to $8^{\text {th }}$ grade of elementary school took part, fourteen students were males and six females, aged between 9 and 13-year old. The students were divided in two groups, with GI composed by ten students with interdisciplinary diagnosis of ADHD and Gll composed of ten students without behavioral disorders and/or learning disorder, paired with GI according to age and grade level. The used procedure was the application of a protocol with tasks of metalinguistic awareness and reading of real words and non-words. Results: there was statistically significant difference between groups in the tasks of syllabic and phonemic manipulation, as well as in tasks of reading real words related to the reading of regular words and irregular words, but also for non-words. Conclusion: the students with Attention Deficit Hyperactivity Disorder showed lower performance in tasks considered to be more complex, such as manipulating syllables and phonemes and reading irregular words, requiring retention, analysis and information retrieval. The difficulties showed in these skills by students with Attention Deficit Hyperactivity Disorder cannot be attributed to a primary deficit, but as a secondary phenomenon to inattention that interferes directly in their performance.

KEYWORDS: Attention Deficit Disorder with Hyperactivity; Reading; Learning; Linguistics; Education

\section{REFERÊNCIAS}

1. Pedrero-Pérez EJ, Ruiz-Sánchez de León JM, Rojo-Mota G, Llanero-Luque M, Puerta-García C. Prevalencia del trastorno por déficit de atención/ hiperactividad en adictos a sustancias: del cribado al diagnóstico. Rev Neurol. 2011; 52: 331-40.

2. Cornelio-Nieto JO, Borbolla-Sala ME, GallegosDimas A. Alteraciones electroencefalográficas en niños con trastorno por déficit de atención/ hiperactividad. Rev Neurol. 2011; 52 (Supl 1): 97-101.

3. Rohde LA, Mattos P. Princípios e práticas em TDAH. Porto Alegre: Artmed; 2003.

4. Pinheiro FH, Lourenceti MD, Santos LCA. Transtorno do déficit de Atenção e Hiperatividade. In: Capellini SA, Germano GD, Cunha VLO. Transtorno de aprendizagem e transtornos de Atenção. Editora: Pulso; 2010. P. 21-34.

5. Cardo E, Servera M, Vidal C, De Azua B, Redondo $M$, Riutort $L$, et al. Influencia de los diferentes criterios diagnósticos y la cultura en la prevalencia del trastorno por déficit de atención/hiperactividad. Rev Neurol. 2011; 52 (Supl 1): 109-17.

6. Martin Fernandez-Mayoralas D, FernandezJaen A, Garcia-Segura JM, Quinones-Tapia D. Neuroimagen en el trastorno por deficit de atencion /hiperactividad. Rev Neurol. 2010; 50 (Supl 3): 125-33.

7. Silveira DC, Passos LMA, Santos PC, Chiapetta ALM. Avaliação da fluência verbal em crianças com transtorno da falta de atenção com hiperatividade: Um estudo comparativo. Rev CEFAC. 2009; 11(2): 208-16.

8. Jou G, Amaral B, Riota C. Transtorno de Déficit de Atenção e Hiperatividade: Um Olhar no Ensino Fundamental. Psicol Reflex Crit. 2010; 23(1):29-36.

9. Barkley RA. Transtorno de Déficit de Atenção/ Hiperatividade - Manual para Diagnóstico e Tratamento. Porto Alegre: Artmed; 2008.

10. Ygual-Fernández A, Cervera-Mérida JF, Baixauli-Fortea I, Meliá-De Alba A. Protocolo de observación del lenguaje para maestros de educación infantil. Rev Neurol. 2011; 52 (Supl 1): 127-34.

11. Asberg J, Dahlgren S, Sandberg, AD. Basic reading skills in high-functioning Swedish children with autism spectrum disorders or attention disorder. Res Autism Spect Dis. 2008; 2: 95-109.

12. Daley D, Birchwood J. ADHD and academic performance: why does ADHD impact on academic performance and what can be done to support ADHD children in the classroom? Ch C Healt Devel. 2010; 36 (4): 455-64.

13. Rohde LA, Halpern R. Transtorno do deficit de atenção/hiperatividade: atualização. J Ped. 2004; 80(2): 61-70.

14. Vasquerio-Madrid J, Estévez-Dias F, Dias-Maílo I. Revisión del modelo de alerta e intervención psicolinguística en el transtorno por déficit de atención e hiperactividad. Rev Neurol. 2006; 42(2): 53-61. 
15. Ettlin T, Kischka U. Beside frontal lobe testing. The frontal lobe score. In: Miller BL, Cummings JL. The human frontal lobes. New York: The Guilford Press; 1998. p. 233-46.

16. van de Voorde $S$, Roeyers $H$, Wiersema JR. Error monitoring in children with ADHD or reading disorders: An event-related potential study. Biol Psychol 2010; 84: 176-85.

17. Castellanos FX, Tannok R. Neurosciece of attention deficit/hyperactivity disorder: the search for endophenotypes. Neurosc. 2002; 3: 617-28.

18. Ygual-Fernández A, Miranda-Casas A, CerveraMérida JF. Dificultades en las dimensiones de forma y contenido del lenguaje en los niños con trastorno por déficit de atención con hiperactividad. Rev Neurol Clin. 2000; 1: 193-202.

19. Capellini SA, Ferreira TL, Salgado CA, Ciasca $\mathrm{SM}$. Desempenho de escolares bons leitores, com dislexia e com transtorno do déficit de atenção e hiperatividade em nomeação automática rápida. Rev Soc Bras Fonoaudiol. 2007; 12(2): 114-9.

20. Lobo PAS, Lima LAM. Comparação do desempenho em leitura de palavras de crianças com e sem transtorno de déficit de atenção/ hiperatividade. Rev CEFAC. 2008; 10 (4): 471-83.

21. Silva C, Capellini SA. Eficácia do programa de remediação fonológica e leitura no distúrbio de aprendizagem. Pró-Fono Rev Atual Cient. 2010; 22(2): 131-8.

22. Miranda-Casas A, Fernández MI, Robledo $P$, García-Castellar R. Comprensión de textos de estudiantes con trastorno por déficit de atención/ hiperactividad: ¿qué papel desempeñan las funciones ejecutivas? Rev Neurol. 2010; 50 (Supl 3): $\$ 135-42$.

23. DSM-IVTM. Manual Diagnóstico e Estatístico de Transtornos Mentais. Porto Alegre: Artes Médicas; 2000.
24. Índice de Desenvolvimento Socioeconômico (IDESE). Fundação de Economia e Estatística (FEE) [homepage na internet]. Porto Alegre: 2003. [acesso em ago 2010]. Disponível: http://www.fee. tche.br/sitefee/pt/content/estatisticas/pg_idese.php 25. Cunha VLO, Capellini SA. PROHMELE - Provas de habilidades metalinguísticas e de leitura. Rio de Janeiro: Revinter; 2009.

26. Scliar-Cabral L. Princípios do sistema alfabético do português do Brasil. São Paulo: Contexto; 2003. 27. Chard, DJ, Stoolmiller, M, Harn, BA, Wanzek, J, Vaughn, S, Linan-Thompson, S, Kame'enui, EJ. Predicting reading success in a multilevel schoolwide reading model. J Learn Disabil. 2008; 41 (2): 174-88.

28. Mulas F, Etchepareborda MC, Abad-Mas L, Díaz-Lucero A. Trastornos neuropsicológicos de los adolescentes afectos de trastorno por déficit de atención con hiperactividad. Rev Neurol. 2006; 43(1): 71-81.

29. Cavadas M, Pereira LD, Mattos P. Efeito do metilfenidato no processamento auditivo em crianças e adolescentes com transtorno do déficit de atenção/hiperatividade. Arq Neuro-Psiquiatr. 2007; 65(1): 138-43.

30. Abdo AGR, Murphy CFB, Schochat E. Habilidades auditivas em crianças com dislexia e transtorno do déficit de atenção e hiperatividade. Pró-Fono Rev Atual Cient. 2010; 22(1): 25-30.

31. Nunes C, Frota S, Mousinho R. Consciência fonológica e o processo de aprendizagem de leitura e escrita: implicações teóricas para o embasamento da prática fonoaudiológica. Rev CEFAC. 2009; 11(2): 207-12.

32. Germano GD, Pinheiro FH, Capellini SA. Desempenho de escolares com dislexia do desenvolvimento em tarefas metalinguísticas e silábicas. Rev CEFAC. 2009; 11(2): 213-20.

http://dx.doi.org/10.1590/S1516-18462012005000003

RECEBIDO EM: 12/04/2011

ACEITO EM: 16/06/2011

Endereço para correspondência:

Vera Lúcia Orlandi Cunha

Rua Clóvis de Camargo Bueno, 218 - Centro

Palmital - SP

CEP: 19970-000

E-mail: verafono@uol.com.br 